\title{
Near-coincidence point results in metric interval space and hyperspace via simulation functions
}

\author{
Misbah Ullah', Muhammad Sarwar', Hasib Khan ${ }^{2 *}$, Thabet Abdeljawad ${ }^{3,4,5 *}$ and Aziz Khan ${ }^{3}$
}

\section{"Correspondence:}

hasibkhan13@yahoo.com;

tabdeljawad@psu.edu.sa

${ }^{2}$ Department of Mathematics,

Shaheed Benazir Bhutto University

Sheringal, Dir Upper, Pakistan

${ }^{3}$ Department of Mathematics and

General Sciences, Prince Sultan

University, Riyadh, Saudi Arabia

Full list of author information is

available at the end of the article

\begin{abstract}
Recently, Wu (Mathematics 6(11):219, 2018; Mathematics 6(6):90, 2018) introduced the concept of a near-fixed point and established some results on near fixed points in a metric interval space and hyperspace. Motivated by these papers, we studied the near-coincidence point theorem in these spaces via a simulation function. To show the authenticity of the established results and definitions, we also provide some examples.
\end{abstract}

Keywords: Near-fixed point; Metric interval space; Hyperspace; Null set; Simulation function; Coincidence point

\section{Introduction and preliminaries}

In mathematics, fixed point theory plays the role of bridge between pure and applied mathematics. Therefore this field has great importance among the branches of pure mathematics and especially in nonlinear analysis. It has many applications to the existence of a solution of a nonlinear system; see, for example, the recent works [3-9] and many more. Metric fixed point theory is the celebrated area in fixed point theory based on the Banach contraction principle (BCP). This principle is given by Banach [10]. After him, this principle was generalized in different forms. This principle is studied in different structures such as dislocated quasimetric spaces [11], cone metric spaces [12], generalized metric spaces [13] and so on. On the other side, the contraction condition is modified in different forms such as the Kannan contraction condition, Chatterjee contraction condition. For details, see [14-16]. Sarwar [17] studied fixed point theorems under rational-type contractions in the setting of complex-valued metric spaces. These results generalized some important results in the present literature. De la Sen [18] used a new approach of $(s-q)$-graphic contraction in $b$-metric-like spaces. These results generalize and improve several approaches in the existing literature by using this new approach for the proof that a Picard sequence is a Cauchy one.

Recently, Khojasteh [19] introduced the concept of a simulation function $\zeta:[0,+\infty) \times$ $[0,+\infty) \longrightarrow \mathbb{R}$ and the concept of $Z$-contraction, which modifies the contraction condition in the Banach contraction principle. Using a simulation function, he proved some

(c) The Author(s) 2020. This article is licensed under a Creative Commons Attribution 4.0 International License, which permits use sharing, adaptation, distribution and reproduction in any medium or format, as long as you give appropriate credit to the original author(s) and the source, provide a link to the Creative Commons licence, and indicate if changes were made. The images or other third party material in this article are included in the article's Creative Commons licence, unless indicated otherwise in a credit line to the material. If material is not included in the article's Creative Commons licence and your intended use is not permitted by statutory regulation or exceeds the permitted use, you will need to obtain permission directly from the copyright holder. To view a copy of this licence, visit http://creativecommons.org/licenses/by/4.0/. 
fixed-point results. Then Hiero et al. [20,21] extended the stated concept of a simulation function given by Khojasteh and investigated some coincidence point results. Argubi [22] used this concept to study the results on coincidence and common fixed point in partially ordered metric space. Alharbi [23] combined the concept of a simulation function with admissible function to generalize some existing results in the related literature. Chanda [24] surveyed many of the recent works related with simulation functions and $Z$-contractions in the existing literature after the publication of Khojasteh et al. Recently, Alsubaie, Alqahtani, and Karapinar [25] proved some interesting results on common fixed points in metric spaces via a simulation function. In 2019, Alqathani and Karapinar [26] introduced the concept of a bilateral contraction, which combines the ideas of Ćirić-type contraction and Caristi-type contraction with the help of simulation function in complete metric spaces. Alghamdi [27] studied common fixed point results in the setting of $b$-metric space via extended $Z$-contraction with respect to a $\psi$-simulation function. His work evaluated and merged as-scattered-as-possible results in fixed point theory from general framework. Karapinar and Agarwal [28] introduced the concept of an interpolative Rus-Reich-Ćirićtype $Z$-contraction in the setting of a complete metric space.

Recently, $\mathrm{Wu}[1,2]$ raised the idea about near-fixed points in metric interval spaces and hyperspaces. He studied some results on near-fixed points in metric interval spaces and hyperspaces. Due to the nonexistence of the inverse of each element, the metric interval spaces and hyperspaces are not conventional metric spaces and normed spaces, respectively. For more detail of interval spaces, we refer the reader to $[29,30]$

Inspired by the works $[19,25-28,31,32]$, we study near-coincidence points in metric interval spaces and hyperspaces via simulation functions. We also provide some examples.

\section{Preliminaries}

We state some basic definitions and fundamental results in this framework.

\subsection{Interval space}

Let $I$ be the collection of all closed bounded intervals $[l, u]$, where $l, u \in \mathbb{R}$ and $l \leq u$; we consider $l \in \mathbb{R}$ as the element $[l, l] \in I$.

The addition and the scaler multiplication are defined as

$$
\begin{aligned}
& {[l, u] \oplus[x, y]=[l+x, u+y],} \\
& k[l, u]= \begin{cases}{[k l, k u],} & k \geq 0, \\
{[k u, k l],} & k<0 .\end{cases}
\end{aligned}
$$

Under these two operation, the inverse of any nondegenerate closed interval does not exist in $I$, so $I$ cannot form the conventional metric space. Clearly, we can see that the additive identity in $I$ is $[0,0]$. However, for $[l, u] \in I$, the subtraction $[l, u] \ominus[l, u]=[l, u] \oplus[-u,-l]=$ $[l-u, u-l]$ does not give a zero element. So the element $[l, u]$ has no inverse element (for more detail, see [1]).

\subsection{Null set}

The null set is defined as

$$
\Omega=\{[t, u] \ominus[t, u]:[t, u] \text { is an element of } I\}
$$


or

$$
\Omega=\{[-\alpha, \alpha] ; \alpha \geq 0\}
$$

In the interval spaces, the following observations are remarkable:

- The distributive law in $I$ is not true in general.

$$
(\alpha+\beta)[t, u] \neq \alpha[t, u] \oplus \beta[t, u] \quad \text { for any }[t, u] \in I \text { and } \alpha, \beta \in \mathbb{R} \text {. }
$$

- The distributivity holds for scaler addition if both scalers are positive or both are negative, that is,

$$
(\alpha+\beta)[t, u]=\alpha[t, u] \oplus \beta[t, u] \quad \text { for any }[t, u] \in I, \alpha, \beta>0 \text {, and } \alpha, \beta<0 \text {. }
$$

- For any $[p, q],[r, s],[t, u] \in I$, we have

$$
\begin{aligned}
{[t, u] \ominus([p, q] \oplus[r, s]) } & =[t, u] \ominus[p, q] \ominus[r, s] \\
& =[t, u] \oplus(-[p, q] \oplus(-[r, s])) .
\end{aligned}
$$

- We write $[t, u] \stackrel{\Omega}{=}[r, s]$ iff there exist $\omega_{1}, \omega_{2} \in \Omega$ such that $[t, u] \oplus \omega_{1}=[r, s] \oplus \omega_{2}$.

Clearly, $[t, u]=[r, s]$ implies $[t, u] \stackrel{\Omega}{=}[r, s]$ by taking $\omega_{1}=\omega_{2}=[0,0]$; however, generally, the converse is not true. Under the relation $\stackrel{\Omega}{=}$, for any $[p, q] \in I$, the equivalence class is defined as $\langle[p, q]\rangle=\{[x, y] \in I:[p, q] \stackrel{\Omega}{=}[x, y]\}$ (for more detail, see [1]).

\subsection{Metric interval space}

A metric interval space is a pair $(I, d)$, where $I$ is the collection of all closed bounded intervals in $\mathbb{R}$ with the null set $\Omega$, and $d$ is a mapping from $I \times I$ to nonnegative real numbers that satisfies the following axioms:

(i) $d([p, q],[r, s])=0$ if and only if $[p, q] \stackrel{\Omega}{=}[r, s]$ for all $[p, q],[r, s] \in I$;

(ii) $d([p, q],[r, s])=d([r, s],[p, q])$ for all $[p, q],[r, s] \in I$;

(iii) $d([p, q],[r, s]) \leq d([p, q],[t, u])+d([t, u],[r, s])$ for all $[p, q],[r, s],[t, u] \in I$;

- If only conditions (ii) and (iii) hold, then the space $(I, d)$ is called a pseudometric interval space.

If the following condition (iv) is satisfied for $d$, then $d$ is said to satisfy the null equalities:

(iv) for any $\omega_{1}, \omega_{2} \in \Omega$ and $[l, u],[x, y] \in I$, the following equalities hold:

(a) $d\left([l, u] \oplus \omega_{1},[x, y] \oplus \omega_{2}\right)=d([l, u],[x, y])$;

(b) $d\left([l, u] \oplus \omega_{1},[x, y]\right)=d([l, u],[x, y])$;

(c) $d\left([l, u],[x, y] \oplus \omega_{2}\right)=d([l, u],[x, y])$.

For more detail, see [1].

Example 2.1 Let $I$ be the collection of all closed bounded intervals in $\mathbb{R}$, and let $d$ be the function from $I \times I$ to $\mathbb{R}^{+}$given by

$$
d([l, u],[x, y])=|(l+u)-(x+y)|
$$

Then $(I, d)$ is a metric interval space with the null equalities satisfied by $d$ (see [1]). 
Definition 2.2 Let $g$ be a self-mapping on a metric interval space $(I, d)$, Then $[l, u] \in I$ is called a near-fixed point of $g$ if $g[l, u] \stackrel{\Omega}{=}[l, u]$ (see [1]).

Definition 2.3 The sequence $\left\{\left[l_{n}, u_{n}\right]\right\}_{n=1}^{+\infty}$ in the space $(I, d)$ is convergent in $I$ if

$$
\lim _{n \rightarrow+\infty} d\left(\left[l_{n}, u_{n}\right],[l, u]\right)=0 \quad \text { for some }[l, u] \in I .
$$

If there exists a point $[x, y]$ such that

$$
\lim _{n \rightarrow+\infty} d\left(\left[l_{n}, u_{n}\right],[x, y]\right)=0, \quad \text { then }[x, y] \in\langle[l, u]\rangle
$$

or

$$
\lim _{n \rightarrow+\infty} d\left(\left[l_{n}, u_{n}\right],[x, y]\right)=0 \quad \text { for all }[x, y] \in\langle[l, u]\rangle .
$$

(For more details see[1].)

Definition 2.4 ([1]) If the limit of the sequence $\left\{\left[l_{n}, u_{n}\right]\right\}_{n=1}^{+\infty}$ is $[l, u]$, then the class $\langle[l, u]\rangle$ is said to be the class limit for $\left\{\left[l_{n}, u_{n}\right]\right\}_{n=1}^{+\infty}$.

We write

$$
\lim _{n \rightarrow+\infty}\left[l_{n}, u_{n}\right]=\langle[l, u]\rangle \quad \text { or } \quad\left[l_{n}, u_{n}\right] \rightarrow\langle[l, u]\rangle .
$$

Definition 2.5 ([1]) Consider the sequence $\left\{\left[l_{n}, u_{n}\right]\right\}_{n=1}^{+\infty}$ in $(I, d)$ such that for any $\epsilon>0$, there exists a natural number $N$ such that $d\left(\left[l_{n}, u_{n}\right],\left[l_{m}, u_{m}\right]\right)<\epsilon$ for all $n, m>N$. Then the sequence is called a Cauchy sequence.

Definition 2.6 ([1]) If every Cauchy sequence is convergent to a point in a subset $J$ of the matric interval space $(I, d)$, then the subset $J$ is said to be complete.

Definition 2.7 (Hierro et al. $[19,20]$ ) A function $S:[0,+\infty) \times[0,+\infty) \rightarrow \mathbb{R}$ is said to be a simulation function if the following conditions holds for $S$ :

$S_{1} . S(0,0)=0$;

$S_{2} . S(r, s)<s-r$ for all $r, s>0$;

$S_{3}$. If $\left\{r_{n}\right\},\left\{s_{n}\right\}$ are two sequences in $(0,+\infty)$ such that $\lim _{n \rightarrow+\infty} r_{n}=\lim _{n \rightarrow+\infty} s_{n}>0$ and $r_{n}<s_{n}$ for all $n \in \mathbb{N}$, then

$$
\lim _{n \rightarrow+\infty} \sup S\left(r_{n}, s_{n}\right)<0
$$

By $S_{2}$ we clearly can say that a simulation function must satisfy

$$
S(l, l)<0 \text {. }
$$

Some examples of simulation functions are:

i. $S(x, y)=\phi(y)-\psi(x)$ for all $x, y \in[0,+\infty)$, where $\phi$ and $\psi$ are continuous function on $[0,+\infty)$ such that $\psi(x)=\phi(x)$ iff $x=0$ and $\psi(x)<x \leq \phi(x)$ for all $x>0$.

Particularly, if we take $\psi(y)=\lambda y$ and $\phi(x)=x$, then $S(x, y)=\lambda y-x$. 
ii. $S(x, y)=y-\phi(y)-x$ for all $x, y \in[0,+\infty)$, where $\phi$ is a continuous function on $[0,+\infty)$ such that $\phi(x)=0$ iff $x=0$ (see [19], Example 2.2).

iii. $S(x, y)=y \psi(y)-x$ for all $x, y \in[0,+\infty)$, where $\psi$ is a mapping such that $\lim _{x \rightarrow r^{+}} \psi(t)<1$ for all $r>0$ [20].

iv. $S(x, y)=\eta(y)-x$ for all $x, y \in[0,+\infty)$, where $\eta$ is a function which is upper semicontinuous, and $\eta(x)<x$ for all $x>0$, and $\eta(0)=0$ [20].

\subsection{Hyperspace}

Let $S(V)$ be the collection of all nonempty convex subsets of $V$, where $V$ is a topological vector space. The two binary operations of addition and scalar multiplication are defined as follows:

$$
\begin{aligned}
& U \oplus U^{*}=\left\{u_{1}+u_{2} ; u_{1} \in U \text { and } u_{2} \in U^{*}\right\} \\
& \mu U=\{\mu u ; u \in U\} .
\end{aligned}
$$

The subtraction is defined by

$$
U \ominus U^{*}=U \oplus\left(-U^{*}\right)=\left\{a-b ; a \in U \text { and } b \in U^{*}\right\} .
$$

Clearly, if $0_{V}$ is the zero element in $V$, then $\left\{0_{V}\right\}$ is the zero element in $S(V)$ because $\left\{0_{V}\right\} \oplus U=U$.

Here in $S(V)$ the inverse does not exist for a nonempty and nonsingleton set, that is, if $\Phi \neq U \neq\left\{0_{V}\right\}$, then $U \ominus U$ is not the zero element of $S(V)$, and so it cannot be a conventional vector space (for more detail, see [2]).

\subsection{Null set}

The null set is defined as $\Omega=\{U \ominus U ; U \in S(V)\}$. This set can be regarded as the zero element of $S(V)$ (see [2]).

\section{Remark 2.8 ([2])}

$1(U \oplus V) \oplus W=U \oplus(V \oplus W)$.

$2 \lambda(U \oplus V)=\lambda U \oplus \lambda V$ for $\lambda \in \mathbb{R}$.

$3 \lambda_{1}\left(\lambda_{2} U\right)=\left(\lambda_{1} \lambda_{2}\right) U$ for $\lambda_{1} \lambda_{2} \in \mathbb{R}$.

4. If $U$ is a convex subset of $V$ and $\lambda_{1}$ and $\lambda_{2}$ have the same sign, then

$$
\left(\lambda_{1}+\lambda_{2}\right) U=\lambda_{1} U \oplus \lambda_{2} U \text {. }
$$

Proposition 2.9 ([2]) For the null set $\Omega$, we have:

i $\left\{0_{V}\right\}=0_{S(v)}$ belongs to the null set.

ii If $\omega \in \Omega$, then $-\omega=\omega$.

iii $\mu \Omega=\Omega$ for $\mu \in \mathbb{R}$ where $\mu \neq 0$.

iv If $\omega_{1}, \omega_{1} \in \mathbb{R}$, then $\omega_{1} \oplus \omega_{2} \in \Omega$.

Definition 2.10 ([2]) For any $U, U^{*} \in S(V)$, we write $U \stackrel{\Omega}{=} U^{*}$ if there exist $\omega_{1}, \omega_{2} \in \Omega$ such that $U \oplus \omega_{1}=U^{*} \oplus \omega_{2}$. The sets $U$ and $U^{*}$ are said to be almost identical. Clearly, $U=U^{*}$ implies $U \stackrel{\Omega}{=} U^{*}$

If $U, U^{*}$, and $W$ are not a singleton set, then $U \ominus U^{*}=W$ implies $U \stackrel{\Omega}{=} U^{*} \oplus W$. 


\subsection{Normed hyperspace}

Let $V$ be a vector space, and let $S(V)$ be the collection of all nonempty convex subsets of $V$. Then $S(V)$ is called a normed hyperspace if there exists a length function $\|\cdot\|: S(V) \rightarrow \mathbb{R}$ satisfying the following axioms:

(i) $\|a U\|=|a|\|U\|$ for all $U \in S(V)$ and $a \in \mathbb{F}$.

(ii) $\left\|U \oplus U^{*}\right\| \leq\|U\|+\left\|U^{*}\right\|$ for all $U, U^{*} \in S(V)$.

(iii) $\|U\|=0$ implies $U \in \Omega$.

If condition (iii) is replaced by $\|U\|=0$ iff $U \in \Omega$, then we say that $\|\cdot\|$ satisfies the null condition (for more detail, see [2]).

Example 2.11 ([2]) Consider a conventional norm space $V$ with conventional norm $\|\cdot\|_{V}$. Let $S(V)$ be the collection of all nonempty convex subsets of $V$. Then the norm defined on $S(V)$ is given as

$$
\|U\|=\sup _{u \in U}\|u\|_{V}
$$

Proposition $2.12([2])$ Let $(S(V),\|\cdot\|)$ be a pseudoseminormed hyperspace such that the null superinequality holds for $\|\cdot\|$. For any $A, C, B_{1}, B_{2}, \ldots, B_{m} \in S(V)$, we have

$$
\|A \ominus C\| \leq\left\|A \ominus B_{1}\right\|+\left\|B_{1} \ominus B_{2}\right\|+\cdots+\left\|B_{j} \ominus B_{j+1}\right\|+\cdots+\left\|B_{m} \ominus C\right\| .
$$

Proposition 2.13 ([2]) We have:

(i) Let $(S(V),\|\cdot\|)$ be a pseudoseminormed hyperspace such that the null equality holds for $\|\cdot\|$. If $U, W \in S(V)$ are such that $U \stackrel{\Omega}{=} W$, then $\|U\|=\|W\|$.

(ii) Let $(S(V),\|\cdot\|)$ be a pseudonormed hyperspace. If $U, W \in S(V)$ are such that $\|U \ominus W\|=0$, then $U \stackrel{\Omega}{=} W$.

(iii) Let $(S(V),\|\cdot\|)$ be a pseudonormed hyperspace such that the null superinequality and null condition holds for $\|\cdot\|$. If $U, W \in S(V)$ are such that $U \stackrel{\Omega}{=} W$, then $\|U \ominus W\|=0$.

\subsection{Convergent sequence}

Let $(S(V),\|\cdot\|)$ be a pseudoseminormed hyperspace (see [2]). A sequence $\left\{U_{n}\right\}_{n=1}^{+\infty}$ in $S(V)$ is said to converge to $U \in S(V)$ if

$$
\lim _{n \rightarrow+\infty}\left\|U_{n} \ominus U\right\|=0
$$

Proposition 2.14 ([2]) Let $(S(V),\|\cdot\|)$ be a pseudoseminormed hyperspace with the null set $\Omega$.

(i) If the sequence $\left\{U_{n}\right\}_{n=1}^{+\infty}$ converges to both $U$ and $U^{*}$, then $U \stackrel{\Omega}{=} U^{*}$.

(ii) Suppose $\|\cdot\|$ satisfies the null equality. If the sequence $\left\{U_{n}\right\}_{n=1}^{+\infty}$ in $S(V)$ converges to $U \in S(V)$, then for any $U^{*} \in[U]$, the sequence $\left\{U_{n}\right\}_{n=1}^{+\infty}$ converges to $U^{*}$.

Definition $2.15([2])$ Let $(S(V),\|\cdot\|)$ be a pseudoseminormed hyperspace. If the sequence $\left\{U_{n}\right\}_{n=1}^{+\infty}$ in $S(V)$ converges to some $U \in S(V)$, then the equivalence class $[U]$ is called the class limit of $\left\{U_{n}\right\}_{n=1}^{+\infty}$. We write this mathematically as

$$
\lim _{n \rightarrow+\infty} U_{n}=[U] \text { or } \quad U_{n} \rightarrow[U]
$$


Definition $2.16([2])$ Let $(S(V),\|\cdot\|)$ be a pseudoseminormed hyperspace. A sequence $\left\{U_{n}\right\}_{n=1}^{+\infty}$ in $S(V)$ is called a Cauchy sequence if for any $\epsilon>0$, there exists $N \in \mathbb{N}$ such that

$$
\left\|U_{n} \ominus U_{m}\right\|<\epsilon \text { for } m, n>N \text {. }
$$

$S(V)$ is said to be complete if every Cauchy sequence in $S(V)$ is convergent.

Definition $2.17([2])$ A normed hyperspace $(S(V),\|\cdot\|)$ is called a Banach hyperspace if it is complete.

Definition 2.18 ([2]) Let $\Gamma$ be a self-mapping on $(S(V),\|\cdot\|)$. Then a point $U \in S(V)$ is called a near-fixed point of $\Gamma$ if $\Gamma[U] \stackrel{\Omega}{=}[U]$.

\section{Results and discussion}

Definition 3.1 If $G[l, u] \stackrel{\Omega}{=} g[l, u]$ for a point $[l, u] \in I$, then $[l, u]$ is called a nearcoincidence point for $G$ and $g$.

Example 3.2 Let us consider two mappings $G$ and $g$ from $I$ to itself defined by

$$
\begin{aligned}
& G[x, y]=[2 x-2,2 x+2], \\
& g[x, y]=[x-1, y+1] \text { for } x, y \in \mathbb{R} \text { and } x \leq y .
\end{aligned}
$$

Then every point in $\Omega$, that is, $[-k, k]$, where $k \in \mathbb{R}^{+}$, is a near-coincidence point for $G$ and $g$. Particularly, if we take $[-1,1]$, then $G[-1,1]=[-4,4]$ and $g[-1,1]=[-3,3]$, so that $[-4,4] \stackrel{\Omega}{=}[-3,3]$. Hence by definition $[-1,1]$ is a near-coincidence point for $G$ and $g$.

Definition 3.3 Let $G$ and $g$ be mappings from $(I, d)$ to itself. Then $G$ and $g$ are called compatible mappings if

$$
\lim _{n \rightarrow+\infty} d\left(G g\left[l_{n}, u_{n}\right], g G\left[l_{n}, u_{n}\right]\right)=0 .
$$

By taking the functions $G$ and $g$ defined before and any sequence, we can easily verify that these mappings are compatible.

Definition 3.4 Mappings $G$ and $g$ are said to be commuting if $G g[l, u] \stackrel{\Omega}{=} g G[l, u]$ for all $[l, u] \in(I, d)$.

Example 3.5 Let us consider previously defined two mappings $G$ and $g: G[x, y]=[2 x-$ $2,2 x+2]$ and $g[x, y]=[x-1, y+1]$ for $x, y \in \mathbb{R}$ and $x \leq y$. Then for any $[x, y] \in I$, we can easily show that $G g[x, y]=[2 x-4,2 y+4] \stackrel{\Omega}{=}[2 x-3,2 y+3]=g G[x, y]$.

To show that $[2 x-4,2 y+4] \stackrel{\Omega}{=}[2 x-3,2 y+3]$, we can take $\omega_{1}=[0,0]$ and $\omega_{2}=[-1,1]$, and hence

$$
[2 x-4,2 y+4] \oplus[0,0]=[2 x-3,2 y+3] \oplus[-1,1] .
$$

Definition 3.6 A mapping $G$ is called a $\left(Z_{d}, g\right)$-contraction in $(I, d)$ if there is a simulation function $S \in Z$ such that $S(d(G[l, u], G[x, y]), d(g[l, u], g[x, y])) \geq 0$ for all $[l, u],[x, y] \in I$, such that $g[l, u] \stackrel{\Omega}{\neq} g[x, y]$. 
Example 3.7 Let us consider the mappings $G$ and $g$ defined on the metric interval space $I$ by $G[x, y]=[2 x-2,2 x+2]$ and $g[x, y]=[x-1, y+1]$. Take the simulation function as $S(t, s)=\lambda s-t$ where $\lambda \geq 2$. Then $G$ is a $\left(Z_{d}, g\right)$-contraction in $(I, d)$, because $S(d(G[l, u], G[x, y]), d(g[l, u], g[x, y])) \geq 0$ for all $[l, u],[x, y] \in I$.

Definition 3.8 A sequence $\left\{\left[l_{n}, u_{n}\right]\right\}$ is said to be a Picard $(G, g)$ sequence at point $\left[l_{o}, u_{o}\right]$ for the mapping $G$ and $g$ in the metric interval space $(I, d)$ if $g\left(\left[l_{n+1}, u_{n+1}\right]\right) \stackrel{\Omega}{=} G\left(\left[l_{n}, u_{n}\right]\right)$ for all $n \geq 0$. The space $(I, d)$ is said to satisfy the $\operatorname{CLR}_{(G, g)}$ property.

Example 3.9 Consider the previously defined mappings $G$ and $g: G[x, y]=[2 x-2,2 x+2]$ and $g[x, y]=[x-1, y+1]$ for $x, y \in \mathbb{R}$ and $x \leq y$. Then the sequence $\left\{\left[x_{n}, y_{n}\right]\right\}=\left\{\left[2^{n}\left(x_{0}-1\right)+\right.\right.$ $\left.\left.1,2^{n}\left(y_{o}+1\right)-1\right]\right\}$ is a Picard sequence at any point $\left[x_{o}, y_{0}\right]$, that is, $g\left(\left[l_{n+1}, u_{n+1}\right]\right) \stackrel{\Omega}{=} G\left(\left[l_{n}, u_{n}\right]\right)$ for all $n \geq 0$.

Definition 3.10 If $g$ is a mapping over a metric interval space $(I, d)$ with a null set $\Omega$, then $g$ is injective if $g[l, u] \stackrel{\Omega}{=} g\left[l^{\prime}, u^{\prime}\right] \Rightarrow\left[l, u \stackrel{\Omega}{=}^{\prime}\left[l^{\prime}, u^{\prime}\right]\right.$ for all $[l, u],\left[l^{\prime}, u^{\prime}\right] \in I$.

Definition 3.11 A point $U \in S(V)$ is said to be a near-coincidence point for $F$ and $g$ if $F[U] \stackrel{\Omega}{=} g[U]$.

Definition 3.12 Let $F$ and $g$ be self-mappings from $S(V)$ to itself. Then $F$ and $g$ are called compatible mappings if

$$
\lim _{n \rightarrow+\infty}\left\|F g\left[U_{n}\right]-g F\left[U_{n}\right]\right\|=0
$$

Definition 3.13 Mappings $F$ and $g$ are said to be commuting if $F g[U] \stackrel{\Omega}{=} g F[U]$ for all $U \in S(V)$.

Definition 3.14 A mapping $F$ is called a $\left(Z_{\|\cdot\|}, g\right)$-contraction in $S(V)$ if there is a simulation function $\xi \in Z$ such that $\xi\left(\left\|G[U]-G\left[U^{*}\right]\right\|,\left\|g[U]-g\left[U^{*}\right]\right\|\right) \geq 0$ for all $U, U^{*} \in S(V)$ such that $g[U] \stackrel{\Omega}{\neq} g\left[U^{*}\right]$.

Definition 3.15 A sequence $\left\{A_{n}, n \geq 0\right\}$ is called a Picard $(F, g)$ sequence in $S(V)$ on the point $\left[A_{o}\right]$ for the mappings $F$ and $g$ on the hyperspace $S(V)$ if $g\left(A_{n+1}\right) \stackrel{\Omega}{=} F\left(A_{n}\right)$ for all $n \geq 0$. Then the space $S(V)$ is said to satisfy the $\operatorname{CLR}_{(F, g)}$ property.

Theorem 3.16 Let $G$ be a self-mapping that is a $\left(Z_{d}, g\right)$-contraction over the complete metric interval space $(I, d)$. Suppose that there exists a Picard sequence for the mappings $G$ and $g$ at a point $[l, u] \in I$, that is,

$$
g\left[l_{n+1}, u_{n+1}\right] \stackrel{\Omega}{=} G\left[l_{n}, u_{n}\right] \text { for all } n \geq 0
$$

Also, assume that the mappings $G$ and $g$ are continuous and compatible. Then there exists a near-coincidence point for $G$ and $g$.

Proof Either the near-coincidence point is contained in the sequence $\left\{g\left[l_{n}, u_{n}\right]\right\}$, or the sequence $\left\{g\left[l_{n}, u_{n}\right]\right\}$ approaches to the required near-coincidence point. 
Suppose that $\left\{g\left[l_{n}, u_{n}\right]\right\}$ contains no near coincidence point of $G$ and $g$, that is,

$$
g\left[l_{n}, u_{n}\right] \stackrel{\Omega}{\neq} G\left[l_{n}, u_{n}\right] \stackrel{\Omega}{=} g\left[l_{n+1}, u_{n+1}\right] \quad \text { for all } n \geq 0
$$

In such a case,

$$
d\left(g\left[l_{n}, u_{n}\right], g\left[l_{n+1}, u_{n+1}\right]\right)>0 \quad \text { for all } n \geq 0
$$

We will prove the result in three steps.

Step 1

We will show that

$$
\lim _{n \rightarrow+\infty} d\left(g\left[l_{n}, u_{n}\right], g\left[l_{n+1}, u_{n+1}\right]\right)=0 .
$$

Using condition (2) of a simulation function and condition of a $\left(Z_{d}, g\right)$-contraction, we have

$$
\begin{aligned}
0 & \leq S\left(d\left(G\left[l_{n}, u_{n}\right], G\left[l_{n+1}, u_{n+1}\right]\right), d\left(g\left[l_{n}, u_{n}\right], g\left[l_{n+1}, u_{n+1}\right]\right)\right) \\
& =S\left(d\left(g\left[l_{n+1}, u_{n+1}\right], g\left[l_{n+2}, u_{n+2}\right]\right), d\left(g\left[l_{n}, u_{n}\right], g\left[l_{n+1}, u_{n+1}\right]\right)\right) \\
& <d\left(g\left[l_{n}, u_{n}\right], g\left[l_{n+1}, u_{n+1}\right]\right)-d\left(g\left[l_{n+1}, u_{n+1}\right], g\left[l_{n+2}, u_{n+2}\right]\right)
\end{aligned}
$$

which implies that

$$
0<d\left(g\left[l_{n+1}, u_{n+1}\right], g\left[l_{n+2}, u_{n+2}\right]\right)<d\left(g\left[l_{n}, u_{n}\right], g\left[l_{n+1}, u_{n+1}\right]\right) .
$$

Note that the sequence $d\left(g\left[l_{n}, u_{n}\right], g\left[l_{n+1}, u_{n+1}\right]\right)$ is a nonnegative decreasing sequence in $\mathbb{R}$, so it converges to a point $l$, that is,

$$
l=\lim _{n \rightarrow+\infty} d\left(g\left[l_{n}, u_{n}\right], g\left[l_{n+1}, u_{n+1}\right]\right)
$$

We will show that $l=0$. Assume that $l \neq 0$, so $l>0$.

Using $S_{3}$ by taking the sequences

$$
\begin{aligned}
& r_{n}=d\left(g\left[l_{n+1}, u_{n+1}\right], g\left[l_{n+2}, u_{n+2}\right]\right) \\
& \text { and } \\
& s_{n}=\left\{d\left(g\left[l_{n}, u_{n}\right], g\left[l_{n+1}, u_{n+1}\right]\right)\right\},
\end{aligned}
$$

we clearly have

$$
\lim _{n \rightarrow+\infty} r_{n}=\lim _{n \rightarrow+\infty} s_{n}=l>0 \quad \text { and } \quad r_{n}<s_{n} \quad \text { for all } n \in N
$$

So by $S_{3}$ we have $\lim _{n \rightarrow+\infty} \sup \left(S\left(r_{n}, s_{n}\right)\right)<0$, that is,

$$
\lim _{n \rightarrow+\infty} \sup \left(S\left(d\left(g\left[l_{n+1}, u_{n+1}\right], g\left[l_{n+2}, u_{n+2}\right]\right), d\left(g\left[l_{n}, u_{n}\right], g\left[l_{n+1}, u_{n+1}\right]\right)\right)\right)<0
$$


which is a contradiction because

$$
0 \leq S\left(d\left(g\left[l_{n+1}, u_{n+1}\right], g\left[l_{n+2}, u_{n+2}\right]\right), d\left(g\left[l_{n}, u_{n}\right], g\left[l_{n+1}, u_{n+1}\right]\right)\right) .
$$

So our supposition that $l \neq 0$ was wrong, and thus $l=0$. Therefore we have

$$
\lim _{n \rightarrow+\infty} d\left(g\left[l_{n}, u_{n}\right], g\left[l_{n+1}, u_{n+1}\right]\right)=0
$$

\section{Step 2}

We will show that $\left\{g\left[l_{n}, u_{n}\right]\right\}$ is a Cauchy sequence in $(I, d)$. On the contrary, suppose that $g\left[l_{n}, u_{n}\right]$ is not Cauchy. So there exists $\epsilon_{o}>0$ such that for all $N \in \mathbb{N}$, there exist positive integers $m, n$ such that

$$
d\left(g\left[l_{n}, u_{n}\right], g\left[l_{m}, u_{m}\right]\right)>\epsilon_{o}
$$

We can construct two subsequences by giving successive values $g\left[l_{n_{k}}, u_{n_{k}}\right]$ and $g\left[l_{m_{k}}, u_{m_{k}}\right]$ to $N$ such that

$$
n_{o} \leq n(k)<m(k) \quad \text { and } \quad d\left(g\left[l_{n_{k}}, u_{n_{k}}\right], g\left[l_{m_{k}}, u_{m_{k}}\right]\right)>\epsilon_{o} \quad \text { for all } k \in \mathbb{N} \text {. }
$$

Let $m(k)$ be the smallest positive integer $m \in\{n(k), n(k)+1, n(k)+2, \ldots\}$ such that (3.2) holds. Now it is clear that

$$
d\left(g\left[l_{m_{k-1}}, u_{m_{k-1}}\right], g\left[l_{n_{k}}, u_{n_{k}}\right]\right) \leq \epsilon_{o} \quad \text { for all } k \in \mathbb{N},
$$

because $m_{k-1}<m(k)$ and $m(k)$ is the least number for which (3.2) holds.

Also, $m(k)>n(k)$ from (3.2), so $m(k) \geq n(k)+1$ for all $k \in \mathbb{N}$. Now if $m(k)=n(k)+1$, then from (3.1) and (3.2) we have

$$
d\left(g\left[l_{n_{k+1}}, u_{n_{k+1}}\right], g\left[l_{n_{k}}, u_{n_{k}}\right]\right)>\epsilon_{o} \quad \text { for all } k \in \mathbb{N},
$$

since from (3.1) we have

$$
d\left(g\left[l_{n_{k+1}}, u_{n_{k+1}}\right], g\left[l_{n_{k}}, u_{n_{k}}\right]\right)<\epsilon_{o} .
$$

Hence $m(k)=n(k)+1$ is not possible by taking into account (3.1) and (3.2), and therefore we conclude that $m(k) \geq n(k)+2$ for any $k \in \mathbb{N}$.

It follows that $n_{k+1}<m_{k}<m_{k+1}$ for all $k \in \mathbb{N}$. From (3.2) and (3.3) we have

$$
\begin{aligned}
\epsilon_{o} & <d\left(g\left[l_{m_{k}}, u_{m_{k}}\right], g\left[l_{n_{k}}, u_{n_{k}}\right]\right) \\
& \leq d\left(g\left[l_{m_{k}}, u_{m_{k}}\right], g\left[l_{m_{k-1}}, u_{m_{k-1}}\right]\right)+d\left(g\left[l_{m_{k-1}}, u_{m_{k-1}}\right], g\left[l_{n_{k}}, u_{n_{k}}\right]\right) \\
& \leq d\left(g\left[l_{m_{k}}, u_{m_{k}}\right], g\left[l_{m_{k-1}}, u_{m_{k-1}}\right]\right)+\epsilon_{o} \quad \text { for all } k \in \mathbb{N} .
\end{aligned}
$$

Therefore

$$
\lim _{k \rightarrow+\infty} d\left(g\left[l_{m_{k}}, u_{m_{k}}\right], g\left[l_{n_{k}}, u_{n_{k}}\right]\right)=\epsilon_{o}
$$


Also,

$$
\lim _{k \rightarrow+\infty} d\left(g\left[l_{m_{k+1}}, u_{m_{k+1}}\right], g\left[l_{n_{k+1}}, u_{n_{k+1}}\right]\right)=\epsilon_{o} .
$$

As $G$ is a $\left(Z_{d}, g\right)$-contraction associated with $S$, we get

$$
\begin{aligned}
0 & \leq S\left(d\left(G\left[l_{m_{k}}, u_{m_{k}}\right], G\left[l_{n_{k}}, u_{n_{k}}\right]\right), d\left(g\left[l_{m_{k}}, u_{m_{k}}\right], g\left[l_{n_{k}}, u_{n_{k}}\right]\right)\right) \\
& =S\left(d\left(g\left[l_{m_{k+1}}, u_{m_{k+1}}\right], g\left[l_{n_{k+1}}, u_{n_{k+1}}\right]\right), d\left(g\left[l_{m_{k}}, u_{m_{k}}\right], g\left[l_{n_{k}}, u_{n_{k}}\right]\right)\right) \\
& <d\left(g\left[l_{m_{k}}, u_{m_{k}}\right], g\left[l_{n_{k}}, u_{n_{k}}\right]\right)-d\left(g\left[l_{m_{k+1}}, u_{m_{k+1}}\right], g\left[l_{n_{k+1}}, u_{n_{k+1}}\right]\right) .
\end{aligned}
$$

Thus

$$
0<d\left(g\left[l_{m_{k+1}}, u_{m_{k+1}}\right], g\left[l_{n_{k+1}}, u_{n_{k+1}}\right]\right)<d\left(g\left[l_{m_{k}}, u_{m_{k}}\right], g\left[l_{n_{k}}, u_{n_{k}}\right]\right) .
$$

Let

$$
r_{n}=d\left(g\left[l_{m_{k+1}}, u_{m_{k+1}}\right], g\left[l_{n_{k+1}}, u_{n_{k+1}}\right]\right)
$$

and

$$
s_{n}=d\left(g\left[l_{m_{k}}, u_{m_{k}}\right], g\left[l_{n_{k}}, u_{n_{k}}\right]\right) .
$$

Clearly, $r_{n}, s_{n}>0, \lim _{n \rightarrow+\infty} r_{n}=\lim _{n \rightarrow+\infty} s_{n}=\epsilon_{o}$, and $r_{n}<s_{n}$.

So by $S_{3}$

$$
0 \leq \lim _{k \rightarrow+\infty} \sup S\left(d\left(g\left[l_{m_{k+1}}, u_{m_{k+1}}\right], g\left[l_{n_{k+1}}, u_{n_{k+1}}\right]\right), d\left(g\left[l_{m_{k}}, u_{m_{k}}\right], g\left[l_{n_{k}}, u_{n_{k}}\right]\right)\right)<0,
$$

which is a contradiction, and hence $\left\{g\left[l_{n}, u_{n}\right]\right\}$ is a Cauchy sequence in $(I, d)$.

Step 3 Now as we have proved that the sequence $\left\{g\left[l_{n}, u_{n}\right]\right\}$ is Cauchy and $(I, d)$ is complete, this sequence is convergent, so there exists $[l, u] \in I$ such that $g\left[l_{n}, u_{n}\right] \rightarrow[l, u]$. The continuity of $G$ and $g$ implies that $g g\left[l_{n}, u_{n}\right] \rightarrow g\left[l_{n}, u_{n}\right]$ and $G g\left[l_{n}, u_{n}\right] \rightarrow G\left[l_{n}, u_{n}\right]$.

Moreover, since $G$ and $g$ are compatible mappings and the limits of the sequences $G\left[l_{n}, u_{n}\right] \stackrel{\Omega}{=} g\left[l_{n+1}, u_{n+1}\right]$ and $g\left[l_{n}, u_{n}\right]$ coincide, we have

$$
\lim _{n \rightarrow+\infty} d\left(G g\left[l_{n}, u_{n}\right], g G\left[l_{n}, u_{n}\right]\right)=0 .
$$

Consider

$$
\begin{aligned}
d(G[l, u], g[l, u]) & =\lim _{n \rightarrow+\infty} d\left(G g\left[l_{n}, u_{n}\right], g g\left[l_{n+1}, u_{n+1}\right]\right) \\
& =\lim _{n \rightarrow+\infty} d\left(G g\left[l_{n}, u_{n}\right], g G\left[l_{n}, u_{n}\right]\right) \\
& =0 .
\end{aligned}
$$

This implies that

$$
G[l, u] \stackrel{\Omega}{=} g[l, u] .
$$

Hence $[l, u]$ is a near-coincidence point of $G$ and $g$. 
To illustrate the theorem, we consider the following example.

Example 3.17 Let $(I, d)$ be a complete metric interval space, and let $G[x, y]=[2 x-2,2 y+2]$ and $g[x, y]=[x-1, y+1]$ be two self-mappings. Take the sequence $\left\{\left[-\frac{1}{n}, \frac{1}{n}\right]\right\}_{n=1}^{n=+\infty}$ in $I$ and the simulation function $S(t, s)=\lambda s-t$ with $\lambda \geq 2$. As we have proved before, the mappings $G$ and $g$ are compatible, and also $G$ is a $\left(Z_{d}, g\right)$-contraction in $(I, d)$. We will just prove that the sequence $\left\{\left[-\frac{1}{n}, \frac{1}{n}\right]\right\}_{n=1}^{n=+\infty}$ is a Picard sequence. For this, we have to show that

$$
g\left[x_{n+1}, y_{n+1}\right] \stackrel{\Omega}{=} G\left[x_{n}, y_{n}\right],
$$

that is, we have to prove that $\left[-\frac{1}{n+1}-1, \frac{1}{n+1}+1\right] \stackrel{\Omega}{=}\left[-2 \frac{1}{n}-2,2 \frac{1}{n}+2\right]$. Take $\omega_{1}=\left[-\frac{n^{2}+2 n+2}{n(n+1)}\right.$, $\left.\frac{n^{2}+2 n+2}{n(n+1)}\right]$ and $\omega_{2}=[0,0]$. Then clearly

$$
\left[-\frac{1}{n+1}-1, \frac{1}{n+1}+1\right] \oplus \omega_{1}=\left[-2 \frac{1}{n}-2,2 \frac{1}{n}+2\right] \oplus \omega_{2} .
$$

Consequently, we have $g\left[x_{n+1}, y_{n+1}\right] \stackrel{\Omega}{=} G\left[x_{n}, y_{n}\right]$. So the sequence $\left\{\left[-\frac{1}{n}, \frac{1}{n}\right]\right\}_{n=1}^{n=+\infty}$ is a Picard sequence for $G$ and $g$. Hence by the theorem the limit of $g\left[x_{n}, y_{n}\right]=\left[-\frac{1}{n}-1, \frac{1}{n}+1\right]$, which is $[-1,1]$, is a near-coincidence point for $G$ and $g$.

If $G$ and $g$ are commuting, then we have

$$
G g\left[l_{n}, u_{n}\right] \stackrel{\Omega}{=} g G\left[l_{n}, u_{n}\right]
$$

which implies that $G$ and $g$ are compatible, that is,

$$
\lim _{n \rightarrow+\infty} d\left(G g\left[l_{n}, u_{n}\right], g G\left[l_{n}, u_{n}\right]\right)=\lim _{n \rightarrow+\infty} d\left(G g\left[l_{n}, u_{n}\right], G g\left[l_{n}, u_{n}\right]\right)=0 .
$$

So we can state the following corollary.

Corollary 3.18 Let $G$ be a Z-contraction in the complete metric interval space $(I, d)$ and suppose there exists a Picard sequence for the mappings $G$ and $g$ at the point $[l, u] \in I$, that is,

$$
g\left[l_{n+1}, u_{n+1}\right]=G\left[l_{n}, u_{n}\right] \text { for all } n \geq 0 .
$$

Also, assume that the mappings $G$ and $g$ are continuous and commuting. Then there exists a near-coincidence point for $G$ and $g$.

Corollary 3.19 Let $G$ and $g$ be the two self-mappings on the complete metric interval space $(I, d)$ satisfying all the conditions stated in Theorem 3.16. If $[l, u]$ and $[w, x]$ are two nearcoincidence points for $G$ and $g$, then $G[l, u] \stackrel{\Omega}{=} g[l, u] \stackrel{\Omega}{=} g[w, x] \stackrel{\Omega}{=} G[w, x]$. Moreover, if one of the mappings $G$ and $g$ is injective, then there exists a unique class of near-coincidence points.

Proof Let $G$ and $g$ have two near-coincidence points $[l, u]$ and $[w, x]$. Then $G[l, u] \stackrel{\Omega}{=} g[l, u]$ and $G[w, x] \stackrel{\Omega}{=} g[w, x]$. We have to show that $g[l, u] \stackrel{\Omega}{=} g[w, x]$. On the contrary, suppose 
$g[l, u] \stackrel{\Omega}{\neq} g[w, x]$. So $d(g[l, u], g[w, x])>0$. Now as $G$ is a $\left(Z_{d}, g\right)$-contraction, we have

$$
\begin{aligned}
0 & \leq S(d(G[l, u], G[w, x]), d(g[l, u], g[w, x])) \\
& =S(d(g[l, u], g[w, x]), d(g[l, u], g[w, x])),
\end{aligned}
$$

it implies that

$$
S(d(g[l, u], g[w, x]), d(g[l, u], g[w, x]))>0 .
$$

which is contradiction to (2.1). So we have $g[l, u] \stackrel{\Omega}{=} g[w, x]$ and hence $G[l, u] \stackrel{\Omega}{=} g[l, u] \stackrel{\Omega}{=}$ $g[w, x] \stackrel{\Omega}{=} G[w, x]$.

Now for the sake of simplicity we assume that $G$ is injective then we have to show that the near-coincidence point is unique.

Let $[l, u]$ and $[w, x]$ are the two coincidence point of $G$ and $g$. Then by above we have $G[l, u] \stackrel{\Omega}{=} g[l, u] \stackrel{\Omega}{=} g[w, x] \stackrel{\Omega}{=} G[w, x] \Rightarrow G[l, u] \stackrel{\Omega}{=} G[w, x] \Rightarrow[l, u] \stackrel{\Omega}{=}[w, x]$ as $G$ is injective Therefore we have $\langle[l, u]\rangle=\langle[w, x]\rangle$. Hence the class of near-coincidence point is unique.

Corollary 3.20 Let $(I, d)$ be a complete metric interval space and let $G$ and $g$ be self mappings on $(I, d)$ such that the $\operatorname{CLR}(G, g)$ property holds in $I$ and $d(G[l, u], G[w, x]) \leq$ $\lambda d(g[l, u], g[w, x])$ for all $[l, u],[w, x] \in I$ such that $g[l, u] \stackrel{\Omega}{\neq} g[w, x]$ where $\lambda \in[0,1)$, then $G$ and $g$ has a near-coincidence point.

Proof We will show that $G$ is a $Z_{d}$-contraction by taking the simulation function $S \in Z$ defined by $S(l, u)=\lambda u-l$ for all $l, u \in[0,+\infty)$ and $\lambda \in[0,1)$.

Since by the given condition we have

$$
d(G[l, u], G[w, x]) \leq \lambda d(g[l, u], g[w, x]) \quad \text { for all }[l, u],[w, x] \in I,
$$

this implies that

$$
\begin{aligned}
0 & \leq \lambda d(g[l, u], g[w, x])-d(G[l, u], G[w, x]) \\
& \leq S(d(G[l, u], G[w, x]), d(g[l, u], g[w, x])) .
\end{aligned}
$$

The last inequality shows that $G$ is a $Z_{d}$-contraction, so by Theorem 3.16 it has a nearcoincidence point in $I$.

Corollary 3.21 Let $(I, d)$ be a complete metric interval space, and let $G$ and $g$ be selfmappings on I that satisfy the following condition:

$$
d(G[l, u], G[x, y]) \leq d(g[l, u], g[x, y])-\Phi(d(g[l, u], g[x, y])) \quad \text { for all }[l, u],[x, y] \in I,
$$

where $\Phi$ is a lower semicontinuous function on $[0,+\infty)$, and $\Phi^{-1}(0)=0$. Then $G$ and $g$ has a near-coincidence point in $I$.

Proof By taking the simulation function $S \in Z$ defined by

$$
S(l, u)=u-\Phi(u)-l \quad \text { for all } l, u \in[0,+\infty) .
$$


We can show that $G$ is a $Z$-contraction, and hence by Theorem $3.16 G$ and $g$ have a nearcoincidence point.

Lemma 3.22 If $F$ is a $\left(Z_{\|} \|, g\right)$-contraction in the hyperspace $S(V)$ and $U$ and $U^{*}$ are two near-coincidence points of $F$ and $g$, then

$$
F(U) \stackrel{\Omega}{=} g(U) \stackrel{\Omega}{=} g\left(U^{*}\right) \stackrel{\Omega}{=} F\left(U^{*}\right) .
$$

Further, if $F$ or $g$ is injective and if they have a near-coincidence point, then it is unique in the sense of equivalence class.

Proof As $U$ and $U^{*}$ are near-coincidence points for $F$ and $g$, we have

$$
F(U) \stackrel{\Omega}{=} g(U) \quad \text { and } \quad F\left(U^{*}\right) \stackrel{\Omega}{=} g\left(U^{*}\right) .
$$

We have to show that $g(U) \stackrel{\Omega}{=} g\left(U^{*}\right)$. On the contrary, suppose $g(U) \stackrel{\Omega}{\neq} g\left(U^{*}\right)$. Then $\| g(U)-$ $g\left(U^{*}\right) \| \geq 0$. As $F$ is a $\left(Z_{\| \cdot} \|, g\right)$-contraction, we have

$$
\begin{aligned}
0 \leq & \xi\left(\left\|F[U]-F\left[U^{*}\right]\right\|,\left\|g[U]-g\left[U^{*}\right]\right\|\right) \\
= & \xi\left(\left\|g[U]-g\left[U^{*}\right]\right\|,\left\|g[U]-g\left[U^{*}\right]\right\|\right) \\
& \because U \text { and } U^{*} \text { are the near-coincidence points for } F \text { and } g .
\end{aligned}
$$

This implies that $\xi\left(\left\|g[U]-g\left[U^{*}\right]\right\|,\left\|g[U]-g\left[U^{*}\right]\right\|\right) \geq 0$, which is a contradiction to (2.1). So $g(U) \stackrel{\Omega}{=} g\left(U^{*}\right)$, and hence

$$
F(U) \stackrel{\Omega}{=} g(U) \stackrel{\Omega}{=} g\left(U^{*}\right) \stackrel{\Omega}{=} F\left(U^{*}\right)
$$

Further, if $F$ or $g$ is injective and if they have a near-coincidence point, then it is unique in the sense of equivalence class. Let $F$ be injective, and let $U$ and $U^{*}$ be two different near-coincidence points for $F$ and $g$. Then

$$
F[U] \stackrel{\Omega}{=} g[U] \quad \text { and } \quad F\left[U^{*}\right] \stackrel{\Omega}{=} g\left[U^{*}\right]
$$

from which we have

$$
F(U) \stackrel{\Omega}{=} g(U) \stackrel{\Omega}{=} g\left(U^{*}\right) \stackrel{\Omega}{=} F\left(U^{*}\right)
$$

This implies that $F(U) \stackrel{\Omega}{=} F\left(U^{*}\right)$, which in turn implies $U \stackrel{\Omega}{=} U^{*}$ because $F$ is injective. So the near-coincidence point is unique in the sense of equivalence class.

Theorem 3.23 Let $(S(V),\|\cdot\|)$ be a Banach hyperspace such that $\|\cdot\|$ satisfies the null equality, and let $F$ be a $\left(Z_{\|\cdot\|}, g\right)$-contraction. Also, assume that the functions $F$ and $g$ are compatible and continuous and there exists a Picard sequence $\left\{A_{n}\right\}$ for $F$ and $g$. Then $F$ and $g$ have at least one near-coincidence point. 
Proof If the sequence $\left\{g\left[A_{n}\right]\right\}$ contains a near-coincidence, then the is nothing to prove. Suppose $\left\{g\left[A_{n}\right]\right\}$ contains no near-coincidence point, that is,

$$
g\left[A_{n} \stackrel{\Omega}{f}_{=}^{\prime} F\left[A_{n}\right] \stackrel{\Omega}{=} g\left[A_{n+1}\right] \text { for all } n \geq 0 .\right.
$$

Hence $\left\|g\left[A_{n}\right] \ominus g\left[A_{n+1}\right]\right\|>0$. First, we will prove that $\lim _{n \rightarrow+\infty}\left\|g\left[A_{n}\right] \ominus g\left[A_{n+1}\right]\right\|=0$.

By $\xi_{2}$ we have

$$
\begin{aligned}
0 & \leq \xi\left(\left\|F\left[A_{n}\right] \ominus F\left[A_{n+1}\right]\right\|,\left\|g\left[A_{n}\right] \ominus g\left[A_{n+1}\right]\right\|\right) \\
& =\xi\left(\left\|g\left[A_{n+1}\right] \ominus g\left[A_{n+2}\right]\right\|,\left\|g\left[A_{n}\right] \ominus g\left[A_{n+1}\right]\right\|\right) \\
& <\left\|g\left[A_{n}\right] \ominus g\left[A_{n+1}\right]\right\|-\left\|g\left[A_{n+1}\right] \ominus g\left[A_{n+2}\right]\right\|,
\end{aligned}
$$

which implies that

$$
0<\left\|g\left[A_{n+1}\right] \ominus g\left[A_{n+2}\right]\right\|<\left\|g\left[A_{n}\right] \ominus g\left[A_{n+1}\right]\right\| \quad \text { for all } n \geq 0 .
$$

The sequence $\left\{\left\|g\left[A_{n}\right] \ominus g\left[A_{n+1}\right]\right\|\right\}$ is a nonincreasing sequence of nonnegative real numbers, so it is convergent. Let its limit be $p$, that is,

$$
\lim _{n \rightarrow+\infty}\left\|g\left[A_{n}\right] \ominus g\left[A_{n+1}\right]\right\|=0 .
$$

To show that $p=0$, let on the contrary suppose that $p>0$. As the sequences $t_{n}=$ $\left\{\left\|g\left[A_{n+1}\right] \ominus g\left[A_{n+2}\right]\right\|\right\}$ and $s_{n}=\left\{\left\|g\left[A_{n}\right] \ominus g\left[A_{n+1}\right]\right\|\right\}$ have the same limit and $t_{n}<s_{n}$ for all $n \geq 0$, applying $\xi_{3}$ to these sequences, we have

$$
\lim _{n \rightarrow+\infty} \operatorname{Sup} \xi\left(t_{n}, s_{n}\right)<0,
$$

which is a contradiction because

$$
\xi\left(\left\|g\left[A_{n+1}\right] \ominus g\left[A_{n+2}\right]\right\|,\left\|g\left[A_{n}\right] \ominus g\left[A_{n+1}\right]\right\|\right) \geq 0 \quad \text { for all } n \geq 0,
$$

and hence $p=0$.

Step 2. Next, we have to show that $\left\{g\left[A_{n}\right]\right\}$ is a Cauchy sequence. On the contrary, suppose that $\left\{g\left[A_{n}\right]\right\}$ is not a Cauchy sequence. So there exists $\epsilon^{\prime}>0$ such that for all $N \in \mathbb{N}$, there exist positive integers $m, n$ such that

$$
\left\|g\left[A_{n}\right] \ominus g\left[A_{m}\right]\right\| \geq \epsilon_{o}
$$

which leads to two subsequences $\left\{g\left[A_{n_{k}}\right]\right\}$ and $\left\{A_{m_{k}}\right\}$ by taking successive values for $N$ such that $n_{o} \leq n_{k}<m_{k}$ and

$$
\left\|g\left[A_{n_{k}}\right] \ominus g\left[A_{m_{k}}\right]\right\|>\epsilon_{o} \quad \text { for all } k \in N .
$$

Also, by the convergence of the sequence $\left\{g\left[A_{n}\right]\right\}$ we have

$$
\left\|g\left[A_{n}\right] \ominus g\left[A_{n+1}\right]\right\|<\epsilon_{o} \quad \text { for all } n \geq n_{o} .
$$


Let $m_{k}$ be the least among $\left\{n_{k}, n_{k+1}, n_{k+2}, \ldots\right\}$ such that (3.4) holds. Then if we take a smaller value than $m_{k}$, then we will have

$$
\left\|g\left[A_{m_{k-1}}\right] \ominus g\left[A_{n_{k}}\right]\right\| \leq \epsilon_{o} \quad \text { for all } k \in \mathbb{N} \text {. }
$$

Also, we cannot take $m_{k}=n_{k}$ and $m_{k}=n_{k+1}$ because (3.4) becomes zero for $m_{k}=n_{k}$, and for $m_{k}=n_{k+1}$, we have

$$
\left\|g\left[A_{n_{k+1}}\right] \ominus g\left[A_{n_{k}}\right]\right\| \leq \epsilon_{o} \quad \text { for all } k \in N
$$

so $m_{k} \geq n_{k+2}$ for all $k \in N$. From Eqs. (3.5) and (3.7) we have

$$
\begin{aligned}
\epsilon_{o} & <\left\|g\left[A_{m_{k}}\right] \ominus g\left[A_{n_{k}}\right]\right\| \\
& \leq\left\|g\left[A_{m_{k}}\right] \ominus g\left[A_{m_{k-1}}\right]\right\|+\left\|g\left[A_{m_{k-1}}\right] \ominus g\left[A_{n_{k}}\right]\right\| \\
& \leq\left\|g\left[A_{n_{k}}\right] \ominus g\left[A_{m_{k}}\right]\right\|+\epsilon_{o} \quad \because \text { by }(3.4) .
\end{aligned}
$$

As $\lim _{n \rightarrow+\infty}\left\|g\left[A_{n_{k}}\right] \ominus g\left[A_{m_{k}}\right]\right\|=0$, by the inequalities we have

$$
\lim _{n \rightarrow+\infty}\left\|g\left[A_{m_{k}}\right] \ominus g\left[A_{n_{k}}\right]\right\|=\epsilon_{o} .
$$

Similarly,

$$
\lim _{n \rightarrow+\infty}\left\|g\left[A_{m_{k+1}}\right] \ominus g\left[A_{n_{k+1}}\right]\right\|=\epsilon_{o} .
$$

Now as $F$ is a $\left(Z_{\|} \cdot \|, g\right)$-contraction, we have

$$
\begin{aligned}
0 & \leq \xi\left(\left\|G\left[A_{m_{k}}\right] \ominus G\left[A_{n_{k}}\right]\right\|,\left\|g\left[A_{m_{k}}\right]-g\left[A_{n_{k}}\right]\right\|\right) \\
& =\xi\left(\left\|g\left[A_{m_{k+1}}\right] \ominus g\left[A_{n_{k+1}}\right]\right\|,\left\|g\left[A_{m_{k}}\right]-g\left[A_{n_{k}}\right]\right\|\right) \\
& <\left\|g\left[A_{m_{k}}\right]-g\left[A_{n_{k}}\right]\right\|-\left\|G\left[A_{m_{k}}\right] \ominus G\left[A_{n_{k}}\right]\right\|,
\end{aligned}
$$

which implies that

$$
0<\left\|g\left[A_{m_{k+1}}\right] \ominus g\left[A_{n_{k+1}}\right]\right\|<\left\|g\left[A_{m_{k}}\right] \ominus g\left[A_{n_{k}}\right]\right\| \quad \text { for all } k \geq n .
$$

Now from the last inequality consider the two sequences $t_{k}=\left\{\left\|g\left[A_{m_{k+1}}\right] \ominus g\left[A_{n_{k+1}}\right]\right\|\right\}$ and $s_{k}=\left\|g\left[A_{m_{k}}\right] \ominus g\left[A_{n_{k}}\right]\right\|$. As $t_{k}$ and $s_{k}$ have the same limit and $t_{k}<s_{n}$, by applying $\xi_{3}$ we have

$$
\lim _{n \rightarrow+\infty} \operatorname{Sup} \xi\left(t_{k}, s_{k}\right)<0,
$$

which is a contradiction because $\xi\left(t_{k}, s_{k}\right)>0$. Hence $\left\{g\left[A_{n}\right]\right\}$ is a Cauchy sequence.

Step 3. In this step, we will show that the limit point of $\left\{g\left[A_{n}\right]\right\}$ is a near-coincidence point for $F$ and $g$. As the space $(S(V),\|\cdot\|)$ is complete, the sequence converges to some limit, say $A$. As $F$ and $g$ are continuous and compatible and $A$ is the limit of $\left\{g\left[A_{n}\right]\right\}$, we have:

$$
\lim _{n \rightarrow+\infty} g\left[A_{n}\right]=A
$$




$$
\begin{aligned}
& \lim _{n \rightarrow+\infty} g g\left[A_{n}\right]=g[A], \quad \text { and } \\
& \lim _{n \rightarrow+\infty} F g\left[A_{n}\right]=F[A] .
\end{aligned}
$$

Consider

$$
\begin{aligned}
\|G[A] \ominus g[A]\| & =\lim _{n \rightarrow+\infty}\left\|F g\left[A_{n}\right] \ominus g g\left[A_{n+1}\right]\right\| \\
& =\lim _{n \rightarrow+\infty}\left\|F g\left[A_{n}\right] \ominus g F\left[A_{n+1}\right]\right\|=0 \quad \because F \text { and } g \text { are compatible. }
\end{aligned}
$$

Hence $\|F[A] \ominus g[A]\|=0$, which implies $F[A] \stackrel{\Omega}{=} g[A]$. So we have proved that $A$ is a nearcoincidence point of $F$ and $g$.

As commuting of $F$ and $g$ implies compatibility, we have the following corollary.

Corollary 3.24 Let $(S(V),\|\cdot\|)$ be a Banach hyperspace such that $\|\cdot\|$ satisfies the null equality, and let $F$ be a $\left(Z_{\|\cdot\|}, g\right)$-contraction. Also, assume that the functions $F$ and $g$ are commuting and continuous and there exists a Picard sequence $\left\{A_{n}\right\}$ for $F$ and $g$. Then $F$ and $g$ have at least one near-coincidence point.

Corollary 3.25 Let $(S(V),\|\cdot\|)$ be a Banach hyperspace such that $\|\cdot\|$ satisfies the null equality, and let $F$ and $g$ be self-mappings on $S(V)$ such that there exists a Picard sequence for $F$ and $g$ in $S(V)$ and

$$
\left\|F[U] \ominus F\left[U^{*}\right]\right\| \leq \lambda\left\|g[U] \ominus g\left[U^{*}\right]\right\| \quad \text { for all } U, U^{*} \in S(V) \text {, where } \lambda \in[0,1),
$$

such that $g[U] \stackrel{\Omega}{\neq} g\left[U^{*}\right]$. Then $F$ and $g$ have a near-coincidence point.

Proof We will show that $F$ is a $Z$-contraction by taking the simulation function $S \in Z$ defined by $S(u, v)=\lambda v-u$ for all $l, u \in[0,+\infty)$ and $\lambda \in[0,1)$.

According to the given condition, we have

$$
\left\|F[U] \ominus F\left[U^{*}\right]\right\| \leq \lambda\left\|g[U] \ominus g\left[U^{*}\right]\right\| \quad \text { for all } U, U^{*} \in S(V) \text {, where } \lambda \in[0,1),
$$

which implies that

$$
\begin{aligned}
0 & \leq \lambda\left\|g[U] \ominus g\left[U^{*}\right]\right\|-\left\|F[U] \ominus F\left[U^{*}\right]\right\| \\
& \leq S\left(\left\|F[U] \ominus F\left[U^{*}\right]\right\|,\left\|g[U] \ominus g\left[U^{*}\right]\right\|\right) .
\end{aligned}
$$

The last inequality shows that $F$ is a $Z_{d}$-contraction, so by Theorem 3.23 it has a nearcoincidence point in $S(V)$.

Corollary 3.26 Let $(S(V),\|\cdot\|)$ be a Banach hyperspace such that $\|\cdot\|$ satisfies the null equality, and let $F$ and $g$ be self-mappings on $S(V)$ that satisfy the following condition:

$$
\left\|F[U] \ominus F\left[U^{*}\right]\right\| \leq\left\|g[U] \ominus g\left[U^{*}\right]\right\|-\Phi\left\|g[U] \ominus g\left[U^{*}\right]\right\| \quad \text { for all } U, U^{*} \in S(V),
$$


where $\Phi$ is a lower semicontinuous function on $[0,+\infty)$, and $\Phi^{-1}(0)=0$. Then $G$ and $g$ have a near-coincidence point in $S(V)$.

Proof By taking the simulation function $S \in Z$ defined by

$$
S(l, u)=u-\Phi(u)-l \text { for all } l, u \in[0,+\infty)
$$

We can show that $F$ is a $Z$-contraction, and hence by Theorem $3.16 F$ and $g$ have a nearcoincidence point.

\section{Conclusion}

Nowadays, the researchers in the subject area are working to produce more effective and generalized fixed point results. Recently, Wu [1,2] introduced the concept of a near-fixed point and established some results on near-fixed points in metric interval spaces and hyperspaces. Motivated by these papers, we studied the near-coincidence point theorem in these spaces via a simulation function. To illustrate the established results and definitions, we included some examples.

\section{Acknowledgements}

The authors are grateful to the editor and anonymous reviewers for their valuable comments and remarks, which helped to improve this manuscript.

\section{Funding}

There is no funding source for the publication of this paper.

\section{Availability of data and materials}

Not related.

\section{Competing interests}

All the authors have no competing interests.

\section{Authors' contributions}

All authors contributed equally to the writing of this manuscript. All authors read and approved the final version.

\section{Author details}

${ }^{1}$ Department of Mathematics, University of Malakand Dir Lower, Chakdara, Pakistan. ${ }^{2}$ Department of Mathematics, Shaheed Benazir Bhutto University Sheringal, Dir Upper, Pakistan. ${ }^{3}$ Department of Mathematics and General Sciences, Prince Sultan University, Riyadh, Saudi Arabia. ${ }^{4}$ Department of Medical Research, China Medical University, Taichung,

Taiwan. ${ }^{5}$ Department of Computer Science and Infromation Engineering, Asia University, Taichung, Taiwan.

\section{Publisher's Note}

Springer Nature remains neutral with regard to jurisdictional claims in published maps and institutional affiliations.

Received: 2 May 2020 Accepted: 28 May 2020 Published online: 15 June 2020

\section{References}

1. Wu, H.-C.: A new concept of fixed point in metric and normed interval spaces. Mathematics 6(11), 219 (2018). https://doi.org/10.3390/math6110219

2. Wu, H.-C.: Near fixed point theorem in hyperspaces. Mathematics 6(6) 90 (2018). https://doi.org/10.3390/math6060090

3. Khan, H., Khan, A., Jarad, F., Shah, A.: Existence and data dependence theorems for solutions of an ABC-fractional order impulsive system. Chaos Solitons Fractals 131, 109477 (2020)

4. Khan, A., Khan, H., Gómez-Aguilar, J.F., Abdeljawad, T.: Existence and Hyers-Ulam stability for a nonlinear singular fractional differential equations with Mittag-Leffler kernel. Chaos Solitons Fractals 127, 422-427 (2019)

5. Khan, H., Jarad, F., Abdeljawad, T., Khan, A.: A singular ABC-fractional differential equation with $p$-Laplacian operator. Chaos Solitons Fractals 129, 56-61 (2019)

6. Khan, H., Li, Y., Khan, A., Khan, A.: Existence of solution for a fractional-order Lotka-Volterra reaction diffusion model with Mittag-Leffler kernel. Math. Methods Appl. Sci. 42(9), 3377-3387 (2019)

7. Khan, H., Abdeljawad, T., Aslam, M., Khan, R.A., Khan, A.: Existence of positive solution and Hyers-Ulam stability for a nonlinear singular-delay-fractional differential equation. Adv. Differ. Equ. 2019, 104 (2019) 
8. Khan, H., Gómez-Aguilar, J.F., Khan, A., Khan, T.S.: Stability analysis for fractional order advection-reaction diffusion system. Phys. A, Stat. Mech. Appl. 521, 737-751 (2019)

9. Khan, H., Tunç, C., Baleanu, D., Khan, A., Alkhazzan, A.: Inequalities for $n$-class of functions using the Saigo fractional integral operator. Rev. R. Acad. Cienc. Exactas Fís. Nat., Ser. A Mat. 113, 2407-2420 (2019)

10. Banach, S.: Sur les opérations dans les ensembles abstraits et leur applications aux equations intégrales. Fundam. Math. 3, 133-181 (1922)

11. Kohli, M., Shrivastava, R., Sharma, M.: Some results on fixed points theorem in dislocated quasi metric spaces. Int. J. Theor. Appl. Sci. 2(1), 27-28 (2010)

12. Guang, H.L., Xian, Z.: Cone metric spaces and fixed point theorems of contractive mappings. J. Math. Anal. Appl. 332(2), 1468-1476 (2007)

13. Sanodia, P.L., Jaiswal, D., Rajput, S.: Fixed point theorems in G-metric spaces via rational type contractive condition. Int. J. Math. Arch. 3(3), 1292-1296 (2012)

14. Kannan, R.: Some results on fixed points. Bull. Calcutta Math. Soc. 60, 71-78 (1968)

15. Chatterjee, S.K.: Fixed-point theorems. C. R. Acad. Bulgare Sci. 25, 727-730 (1972)

16. Aage, C.T., Salunke, J.N.: Fixed points for weak contractions in G-metric spaces. Appl. Math. E-Notes 12, 23-28 (2012)

17. Sarwar, M., Zada, M.B., Radenovíc, S.: Rational type inequality with applications to Volterra-Hammerstein nonlinear integral equations. Int. J. Nonlinear Sci. Numer. Simul. (2020). https://doi.org/10.1515/ijnsns-2018-0367

18. de la Sen, M., Nikolíc, N., Došenovíc, T., Pavlovíc, M., Radenovíc, S.: Some results on $(s-q)$-graphic contraction mappings in b-metric-like spaces. Mathematics 7, 1190 (2019). https://doi.org/10.3390/math7121190

19. Khojasteh, F., Shukla, S., Radenovíc, S.: A new approach to the study of fixed point theorems via simulation functions. Filomat 29(6), 1189-1194 (2015)

20. Roldan Lopez de Hierro, A.F., Karapinar, E., Roldan Lopez de Hierro, C., Martnez-Moreno, J.: Coincidence point theorems on metric spaces via simulation functions. J. Comput. Appl. Math. 275, 345-355 (2015)

21. Roldan Lopez de Hierro, A.F., Karapinar, E., O'Regan, D.: Coincidence point theorem on quasi metric spaces via simulation functions and application to G-metric spaces. J. Fixed Point Theory Appl. 20, 112 (2018). https://doi.org/10.1007/s11784-018-0582-x

22. Argoubi, H., Samet, B., Vetro, C.: Nonlinear contractions involving simulation functions in a metric space with a partial order. J. Nonlinear Sci. Appl. 8, 1082-1094 (2015)

23. Alharbi, S., Alsulami, H.H., Karapinar, E.: On the power of simulation and admissible functions in metric fixed point theory. J. Funct. Spaces. 2017, Article ID 2068163, 7 pages (2017). https://doi.org/10.1155/2017/2068163

24. Chanda, A., Dey, L.K., Radenovíc, S.: Simulation functions: a survey of recent results. Rev. R. Acad. Cienc. Exactas Fís. Nat., Ser. A Mat. 113, 2923-2957 (2019). https://doi.org/10.1007/s13398-018-0580-2

25. Alsubaie, R., Alqahtani, B., Karapinar, E., Hierro, A.F.R.L.: Extended simulation function via rational expressions. Mathematics 8, 710 (2020)

26. Alqahtani, O., Karapinar, E.: A bilateral contraction via simulation function. Filomat 33(15), 4837-4843 (2019)

27. Alghamdi, M.A., Gulyaz-Ozyurt, S., Karapinar, E.: A note on extended Z-contraction. Mathematics 8, 195 (2020)

28. Agarwal, R.P., Karapinar, E.: Interpolative Rus-Reich-Ciric type contractions via simulation functions. An. Ştiinţ. Univ. 'Ovidius' Constanța, Ser. Mat. 27(3), 137-152 (2019)

29. Moore, R.E.: Interval Analysis. Prentice-Hall, Englewood Cliffs (1966)

30. Wu, H.-C.: Hahn-Banach theorems in nonstandard normed interval spaces. Nonlinear Anal. 72, 469-477 (2010) (4): 2663-2679 (2017)

31. Aydi, H., Karapinar, E., Rakocevic, V:: Nonunique fixed point theorems on $b$-metric spaces via simulation functions. Jordan J. Math. Stat. 12(3), 265-288 (2019)

32. Karapinar, E., Khojasteh, F.: An approach to best proximity points results via simulation functions. J. Fixed Point Theory Appl. 19(3), 1983-1995 (2017). https://doi.org/10.1007/s11784-016-0380-2

\section{Submit your manuscript to a SpringerOpen ${ }^{\circ}$ journal and benefit from:}

- Convenient online submission

- Rigorous peer review

- Open access: articles freely available online

- High visibility within the field

- Retaining the copyright to your article

Submit your next manuscript at $\gg$ springeropen.com 